\title{
Combined finite element and multibody musculoskeletal investigation of a fractured clavicle with reconstruction plate
}

\section{Marie Cronskär, John Rasmussen \& Mats Tinnsten}

To cite this article: Marie Cronskär, John Rasmussen \& Mats Tinnsten (2015) Combined finite element and multibody musculoskeletal investigation of a fractured clavicle with reconstruction plate, Computer Methods in Biomechanics and Biomedical Engineering, 18:7, 740-748, DOI: 10.1080/10255842.2013.845175

To link to this article: https://doi.org/10.1080/10255842.2013.845175

Published online: 24 Oct 2013.

Submit your article to this journal $₫$

Џll Article views: 435

Q View related articles $\sqsubset$

View Crossmark data

Citing articles: 18 View citing articles 


\title{
Combined finite element and multibody musculoskeletal investigation of a fractured clavicle with reconstruction plate
}

\author{
Marie Cronskär $^{\mathrm{a} *}$, John Rasmussen ${ }^{\mathrm{b} 1}$ and Mats Tinnsten ${ }^{\mathrm{a} 2}$ \\ ${ }^{a}$ Department of Technology and Sustainable Development, Mid Sweden University, 83125 Östersund, Sweden; ${ }^{b}$ Department of \\ Mechanical and Manufacturing Engineering, Aalborg University, DK-9000 Aalborg, Denmark
}

(Received 11 September 2012; accepted 12 September 2013)

\begin{abstract}
This paper addresses the evaluation of clavicle fixation devices, by means of computational models. The aim was to develop a method for comparison of stress distribution in various fixation devices, to determine whether the use of multibody musculoskeletal input in such model is applicable and to report the approach. The focus was on realistic loading and the motivation for the work is that the treatment can be enhanced by a better understanding of the loading of the clavicle and fixation device. The method can be used to confirm the strength of customised plates, for optimisation of new plates and to complement experimental studies. A finite element (FE) mesh of the clavicle geometry was created from computed tomography data and imported into the FE solver where the model was subjected to muscle forces and other boundary conditions from a multibody musculoskeletal model performing a typical activity of daily life. A reconstruction plate and screws were also imported into the model. The combination models returned stresses and displacements of plausible magnitudes in all included parts and the result, upon further development and validation, may serve as a design guideline for improved clavicle fixation.
\end{abstract}

Keywords: clavicle; finite element analysis; multibody simulation; bone plate

\section{Introduction}

The treatment of clavicle fractures varies greatly in different countries (Dines et al. 2008). There are differing opinions with regard to the optimal treatment of the various types of fractures. Traditionally, non-operative treatment has been preferred for fresh fractures of the clavicle, with few exceptions. The current research is inconsistent in this aspect. Some studies indicate that nonoperative treatment is preferable (Bostman et al. 1997; Hill et al. 1997; Judd et al. 2009), while others show that the occurrence of malunion, non-union and sequelae is the consequence of non-operative treatment (McKee et al. 2003; Ledger et al. 2005; Alatamimi and McKee 2008). In a study involving around 200 patients Nowak found that displaced fractures, particularly where there were no osseous contact and comminuted fractures, especially with transversally located fragments, were associated with a significantly increased risk of remaining symptoms when treated traditionally (Nowak 2002). Although there are large differences in treatment practice, there are some types of complex fractures and cases of non-union which always require surgery.

The clavicle is an s-shaped tubular bone which forms a part of the complex shoulder girdle and acts like a strut to hold the upper limb away from the body. The muscles attached to the clavicle are the deltoid, trapezius, sternocleidomastoid, pectoral and subclavius which are responsible for the typical displacement seen in midshaft fractures (Dines et al. 2008). Present options for operative treatment are intramedullary devices, reconstruction plates which require shaping during surgery to follow the bone contour and anatomically shaped plates which are precontoured to follow the shape of an average clavicle. The intramedullary device is a less invasive alternative than plating but appears to be inadequate where rotational stiffness is required (Golish et al. 2008; Renfree et al. 2010). Huang et al. (2007) investigated the fit of a pre-contoured plate on 100 pairs of clavicle bones and found that these had a poor fit on $38 \%$ of the female clavicles. The optimum treatment for these types of fractures is still under investigation but a better understanding of the loading of the clavicle and the stresses in the clavicle/plate is needed for the choice of fixation technique and as a basis when developing the fixation devices for clavicle fractures.

Even though the shoulder girdle is exposed to such a complex loading, previous biomechanical studies and plate failure studies on the clavicle use various and often greatly simplified load cases such as axial compression or cantilever bending. There is an inconsistency in those results and, to a large extent, the inconsistency is probably due to the difference in test modes (Taylor et al. 2011). In a recent study by Iannolo et al. (2010), the forces across the clavicle during glenohumeral motion are studied in detail. Taylor et al. also used a more realistic load case in their

*Corresponding author. Email: marie.cronskar@miun.se

(C) 2013 Taylor \& Francis 
biomechanical studies, based on the attached muscles and ligaments, whose forces according to a biomechanical model were converted to section forces and moments across the fracture and used as loads in subsequent sawbone experiments. A mathematical simulation of the early rehabilitation moments on the clavicle was used. It was found that the simulation results corresponded better to experimental results than traditional three-point and cantilever bending (Taylor et al. 2011).

To obtain a realistic load case for the finite element (FE) model, a musculoskeletal model similar to that used in Taylor's study was developed. The difference between the two studies is that Taylor's model focused on section forces and moments in the fracture gap for the purpose of constructing test equipment for the mechanical testing of a clavicle with a fixation plate. In the present study, the model focused on muscle and ligament forces distributed throughout the clavicle for use as boundary conditions for an FE analysis. To the best of the author's knowledge, no FE model including bone with implant and a load case based on muscle and ligament forces has been previously reported in the literature.

The aim of this study was to develop a method for the comparison of different fixation devices for clavicle fractures. In a future work, the model will be further developed and used to compare the stress distribution in customised plates with current commercial plates, in plates with different shape, in different positions and with varying screw joints.

\section{Materials and methods}

\section{Image data collection and bone modelling}

The clavicle bone model was created from a computed tomography (CT) scan of a left, healthy clavicle of a 36-year-old woman. The woman had a non-union fracture on the right clavicle with a large degree of callus formation. A fracture was simulated on a 3D model of the left clavicle and the fracture gap was oriented to approximately reproduce the positioning of the fracture of the right clavicle. The reason for using the CT data on the left clavicle was the presence of callus on the right, which would have to be removed in order to be able to simulate plating on a fresh fracture.

The CT image acquisition was performed using a Light Speed Pro 16 system (GE Medical Systems) and then reconstructed from raw data using a slice thickness of $0.6 \mathrm{~mm}$ and a $512 \times 512$ image matrix resolution in a Bone Plus algorithm. The CT scan was retrieved from the picture archiving and communication system station and anonymised. In the radiotherapy department, a 3D model of the clavicle was reconstructed utilising a GE Advanced Workstation 4.6 (GE Medical Systems). The digital imaging and communications in medicine (DICOM) images were segmented using a dynamic region technique and a Hounsfield value of 102 and above. The model was then sliced at $0.8 \mathrm{~mm}$ reconstruction intervals.

The CT data were imported into the Mimics 14.11 (Materialise, Leuven, Belgium) software for thresholding between the Hounsfield values 111 and 2310 to achieve the outer contours of the bone. Subsequently, an approximation of the inner spongious bone was created based on the grey-scale values of the bone. The dark, spongious part of the bone was marked in every 5th-10th image throughout the bone (Figure 1) and a stereolithography (STL) file of the spongious bone was created from the interpolation of the slices and smoothed in the 3-matic 14.11 (Materialise, Leuven, Belgium) software. A Boolean operation was performed to remove the spongious bone from the bone model in order to create an STL-file which represented only the cortical part of the bone. Hence, the spongious part was left as a cavity without material properties. As the task of the bone is to transfer forces from the muscle and ligaments to the plate and the centre of the bone has small impact on the bending resistance, it was assumed that leaving the cavity without spongious material properties would not have much impact on the stresses in the plate. To confirm this assumption, a comparing stress analysis was carried out. One model contains a solid bone (cortical material properties through all) and one with a cavity which corresponds to the spongious part of the bone. The resulting maximum stresses and displacements in the plate in the solid and hollow models were $481 \mathrm{MPa}, 0$. $57 \mathrm{~mm}$ and $483 \mathrm{MPa}, 0.59 \mathrm{~mm}$, respectively, which means that the resulting stresses would be even smaller comparing the current model used in this study with a model with material properties for trabecular bone in the cavity. Therefore, the authors decided to simplify the bone model by excluding the influence of the spongious part of the bone.

A simulated fracture was created in the Magics 16.0 (Materialise, Leuven, Belgium) software by splitting the bone approximately in the same manner and position as the fracture of the right bone. A fracture gap of $0.5 \mathrm{~mm}$ was created so that the fracture edges could later be remeshed to obtain high-quality triangles, without generating any overlapping triangles between the two parts of the bone. The bone mesh, plate and screws were imported into the Magics software, the plates and screws were oriented in the correct positions and a Boolean operation on the mesh was used to create the screw holes in the bone mesh (see Figure 2).

To achieve a functional mesh for use in the FE simulations, the mesh of the fractured cortical bone was refined using the 3-matic software to meet the requirements for not having any angles less than $25^{\circ}$ and a maximum edge length of $2 \mathrm{~mm}$. A volume mesh was created and exported to the Abaqus 6.11 (Dassault Systemes, Vélizy-Villacoublay, France) input format. 

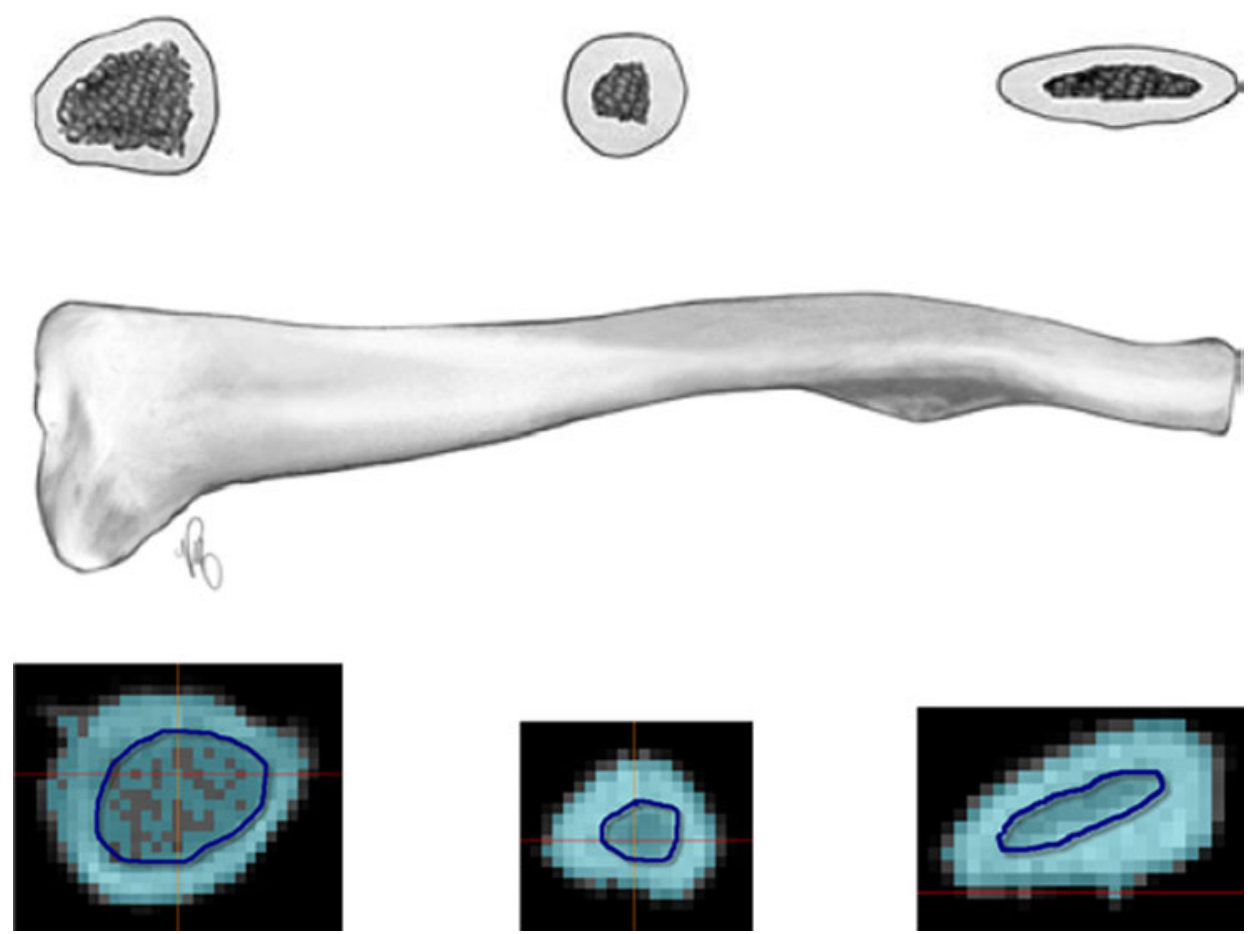

Figure 1. Above: cross section of the various parts of the clavicle (Nowak 2002). Below: markings of the spongious bone, based on grey-scale values, in different parts of the clavicle.

\section{Muscle and ligament forces on the clavicle}

The adapted clavicle geometrical model was imported into the AnyBody Modeling System (AnyBody Technology, Aalborg, Denmark) and subjected to a multibody musculoskeletal analysis based on inverse dynamics assuming a third-order muscle recruitment criterion. For details on the musculoskeletal simulation method, please refer to Damsgaard et al. (2006). The simulations were based on the eating motion, more precisely the static position when holding a cup of tea weighting $0.5 \mathrm{~kg}$, in front of the mouth (see Figure 3).

The reaction forces on the clavicle, derived from the muscles and ligaments were exported from the AnyBody Modeling software as an Extensible Markup Language (xml) file. The Abaqus input mesh and the xml-file of the load case were converted into a new Abaqus input file (inp) using a converter tool included in the AnyBody suite. The input file was then imported into the Abaqus software. The surface areas representing the muscle and ligament attachments were manually adjusted to provide a better representation of the real areas of attachment. The parts of the muscular attachments were later removed where the plate was attached, in accordance with surgical practice (see Figure 4).

\section{Reconstruction plate and screws}

The plate used in this study is a locking compression plate (LCP) Reco-Plate 3.5 straight, 6 holes (Synthes, West Chester,

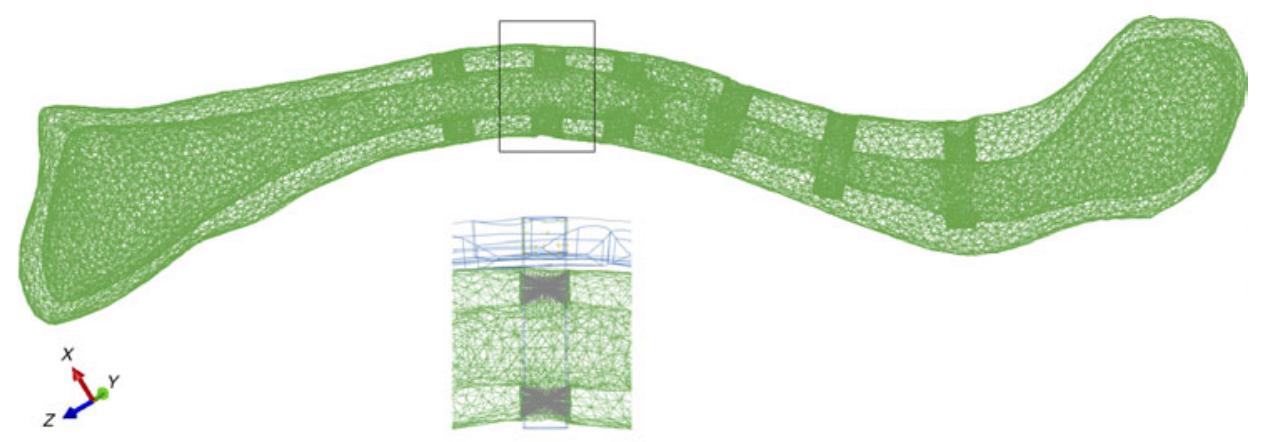

Figure 2. Mesh of the cortical bone with screw holes seen from below. Enlargement with plate, screw and constraints between screw and cortical part of bone mesh. 


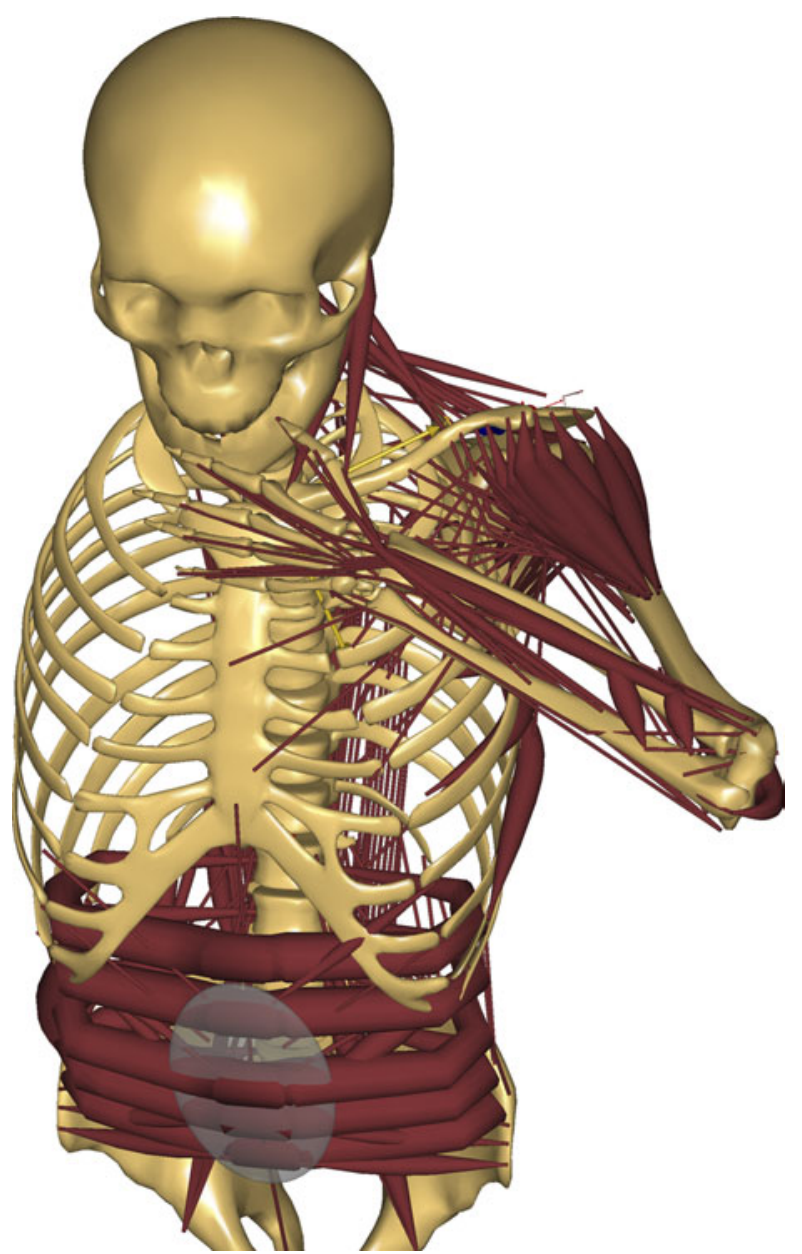

Figure 3. The position of the arm in the biomechanical model.

PA, USA). This is a plate which is sometimes used for the fixation of clavicle non-unions and the plate which was used for fixation in the patient from which the CT data were received for the FE model in this study. The geometry of the LCP Reco-Plate was scanned with a FARO Titanium measurement arm with laser scanner and modelled in Rhinoceros 4.0 (McNeal, Seattle, WA, USA) and Solid Works 2010 (Dassault Systemes, Waltham, MA, USA) using the data from the 3D scan. The screw holes were modelled without threads and countersinks. The plate was modelled as a straight reconstruction plate and later bent in the Solid Works software using the 'flex' command to follow the bone contour. The 3D model of the plate was later imported into the FE model in the universal Initial Graphics Exchange Specification (IGES) file format. The screws were simplified and modelled in the Solid Works software without threads and heads. The focus in this article is on stresses in the plate. The authors have not found any reported problems with high stresses in the screws and the clavicle is a bone with good bone quality not primarily known for screw loosening; hence, the screwsbone-plate interfaces are simplified in this model.

\section{FE model of bone with plate}

The FE analysis program Abaqus 6.11 (Dassault Systemes) was used for the simulation work. Initially, the input file containing the bone mesh with loads from the biomechanical model was imported. In this file, the muscle and ligament forces are modelled as concentrated forces at a point which is coupled (in all 6 degrees of freedom) to a surface representing the muscle or ligament attachment to the bone. The bone mesh was refined from linear (4 nodes) tetrahedral to quadratic (10 nodes) tetrahedral and the total number of elements in the bone mesh was 63,021 (100,045 nodes). The plate and screws were imported as IGES files and later the plate was meshed with 41,681 quadratic tetrahedral elements of type C3D10 (Abaqus) and 66,613 nodes. Each screw was meshed with $\sim 3000$ quadratic tetrahedral elements (5000 nodes). The plate and screws were assembled and oriented to fit the bone mesh. The

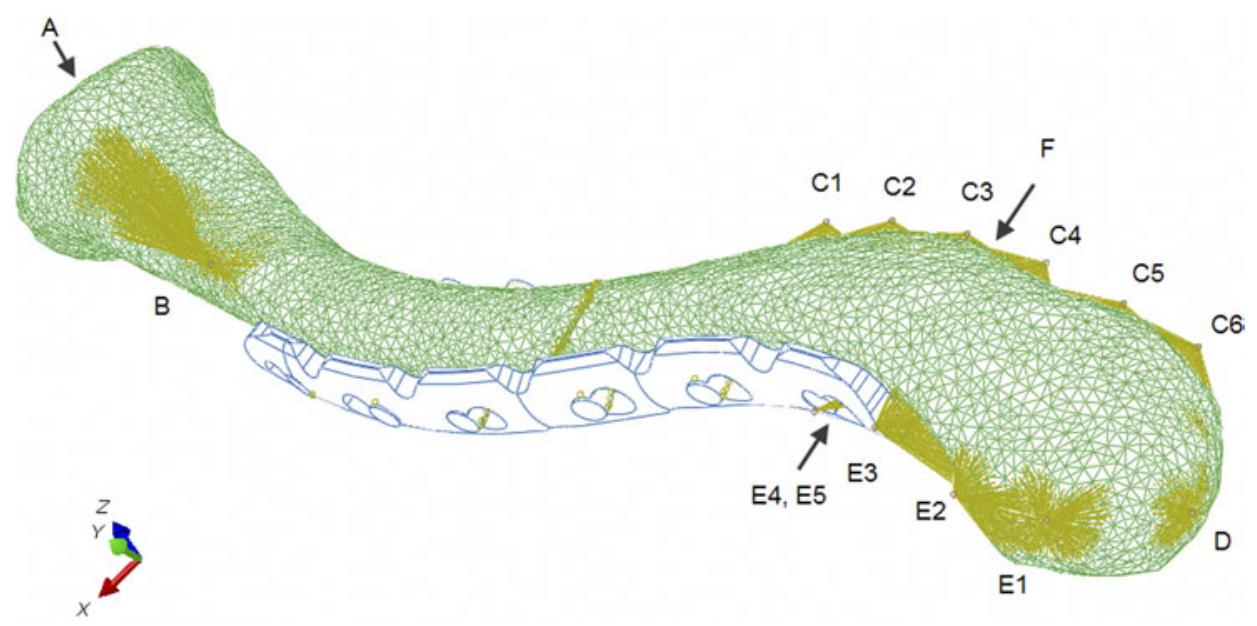

Figure 4. FE model of bone with plate and screws and muscle and ligament attachment areas. For labels A-F, see Table 1. The labels with arrows are muscle and ligament attachments not visible in current view. 
screws were divided into three sections. The upper sections were modelled with coupling constraints to the inner surfaces of the plate holes, simulating locked screws. The two lower sections of the screws were coupled to upper and lower cortical bone segments of the screw holes made in the bone mesh (see Figure 2). A boundary condition, locking all linear translations and the rotation around the axis along the clavicle bone, was assigned to a small surface of the medial end of the bone. At the distal

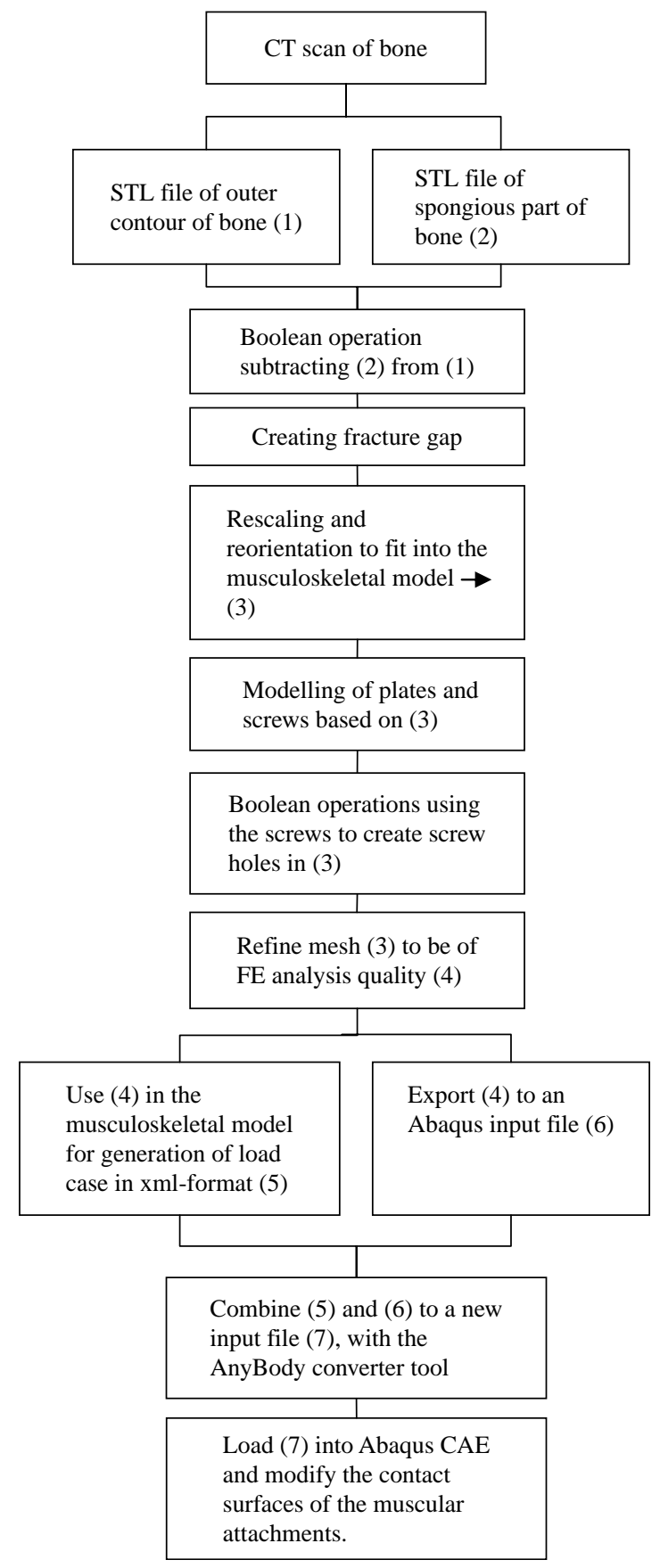

Figure 5. Procedure for combining the bone/implant FE model with a multibody musculoskeletal load case. end, there is a small surface where the linear translations are locked in the transverse directions. The largest reaction force in the boundary conditions was $2.6 \mathrm{~N}$, demonstrating that the muscle and ligament forces are almost in equilibrium. A surface-to-surface contact interaction was modelled in the fracture gap. The FE model is shown in Figure 4.

The material properties used for the cortical bone differ in the literature (Wirtz et al. 2000; Ataç et al. 2009; Pérez et al. 2011; Wong et al. 2011; Kunze et al. 2012). In this case, an anisotropic material was used for the cortical bone with a Young's modulus of $E=18 \mathrm{GPa}$ in the longitudinal direction of the bone and $E=8 \mathrm{GPa}$ in the transversal direction (Kim et al. 2011). The Poisson's ratio was 0.3. The influence of the inner spongious bone is excluded in this model. The plate and screws were assigned as a stainless steel (316 L) material, $E=186.4 \mathrm{GPa}$ and a Poison's ratio of 0.3 (Disegi 2009). The procedure for creating the FE model including the bone mesh generated from CT data and a load case based on a multibody musculoskeletal model generated in the AnyBody software is presented step by step in Figure 5.

\section{Results}

The forces resulting from the musculoskeletal model are shown in Table 1. For muscle and ligament attachments (see Figure 4). For the current arm position, the largest forces come from the conoid ligament, the acromion contact and the deltoid muscle.

The procedure for simulating the stresses in a reconstruction plate placed on a clavicle bone model with a load case based on the muscle and ligament forces was successful and showed plausible results. The maximal Von Mises stress in the middle of the plate was in the order of 150-220 MPa with a maximal stress of $460 \mathrm{MPa}$ on the edge of tapering reconstruction segment (Figure 6). In the stress concentrated areas of the screws, the stresses were around

Table 1. Muscle and ligament forces on the clavicle from the multibody musculoskeletal simulation (for coordinate system and muscle attachment areas, see Figure 4).

\begin{tabular}{lccc}
\hline & \multicolumn{3}{c}{ Muscle force components $(N)$} \\
\cline { 2 - 4 } Muscle/ligament & $F x$ & $F y$ & $F z$ \\
\hline Lig. conoid (F) & -86.8 & -84.8 & -11.5 \\
Lig. trapezoid & 0 & 0 & 0 \\
M. deltoid (E) & 59.2 & -46.8 & -25.8 \\
M. pect. major & 0 & 0 & 0 \\
M. sternocleidomastoid (B) & -4.2 & 14.2 & -1.5 \\
M. trapezius (C) & -2.8 & 22.4 & 30.5 \\
Sternum contact force (A) & 11.1 & 13.4 & -10.1 \\
M. trapezius (C) & -2.8 & 22.4 & 30.5 \\
Sternum contact force (A) & 11.1 & 13.4 & -10.1 \\
Acromion contact force (D) & 23.4 & 86.5 & 18.3 \\
\hline
\end{tabular}




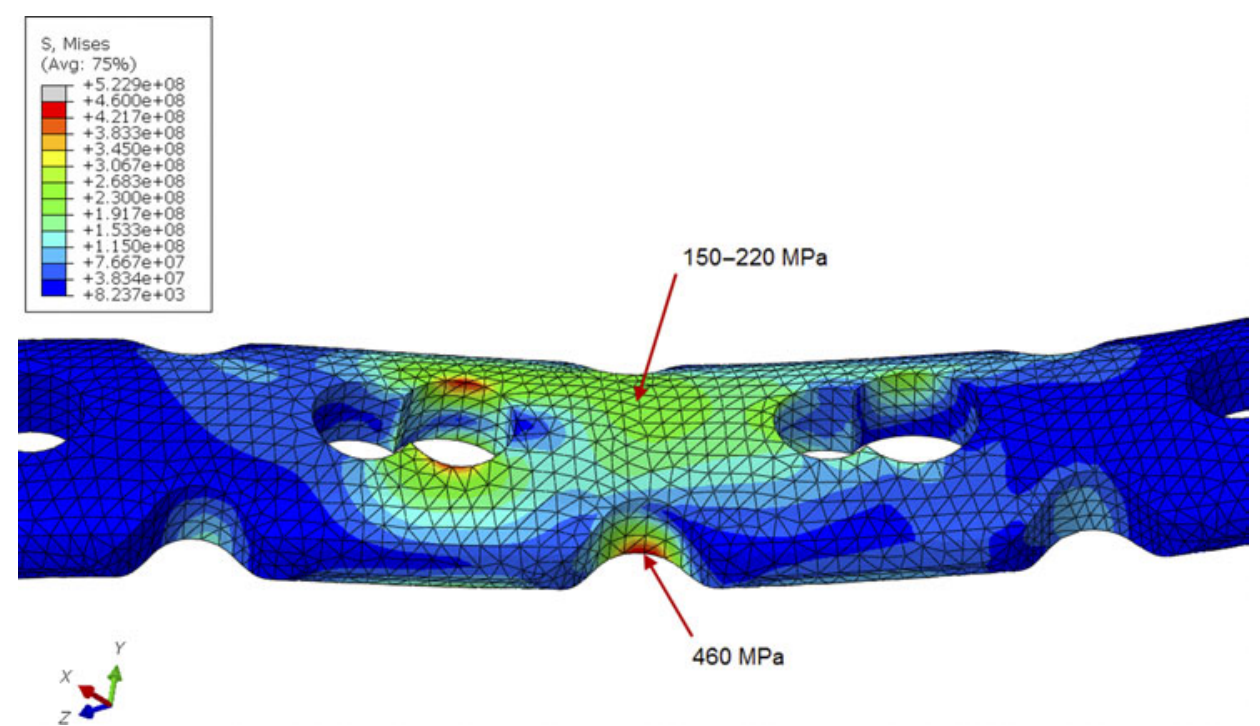

Figure 6. Stresses in the reconstruction plate.

100-200 MPa with a maximum of $330 \mathrm{MPa}$ in the spacing between the plate and bone, in one of the screws in the middle of the plate. The resulting displacement $(U)$ in the plate varies between 0.3 and $0.6 \mathrm{~mm}$ (Figure 7). The fracture is in such a position that, together with a plate in current position, the load case caused by the studied arm position tends to open up the fracture. Hence, the contact interaction in the fracture gap was not activated and the plate is subjected to the entire load. The maximal Von Mises stress in the bone is around $20 \mathrm{MPa}$ (Figure 8). For comparison, a second FE analysis was performed in order to investigate the stress pattern in the bone without fracture and plate. The results are shown in Figure 9.

\section{Discussion}

To our knowledge, this is the first FE study to use a validated musculoskeletal model to estimate realistic loads for a clavicle in an arm position which represents early rehabilitation after clavicle fixation surgery. Previous FE analyses of clavicles mainly concern injury predictions (Astier et al. 2008; Arregui-Dalmases et al. 2010) and the authors have not found any clavicle FE models including implants with which to compare their results. Similar studies with bone and implant but with simplified load cases have been shown to be usable for implant analysis in other parts of the body, for example, on the proximal humerus (Young et al. 2010), the maxilla (Ataç et al. 2008;
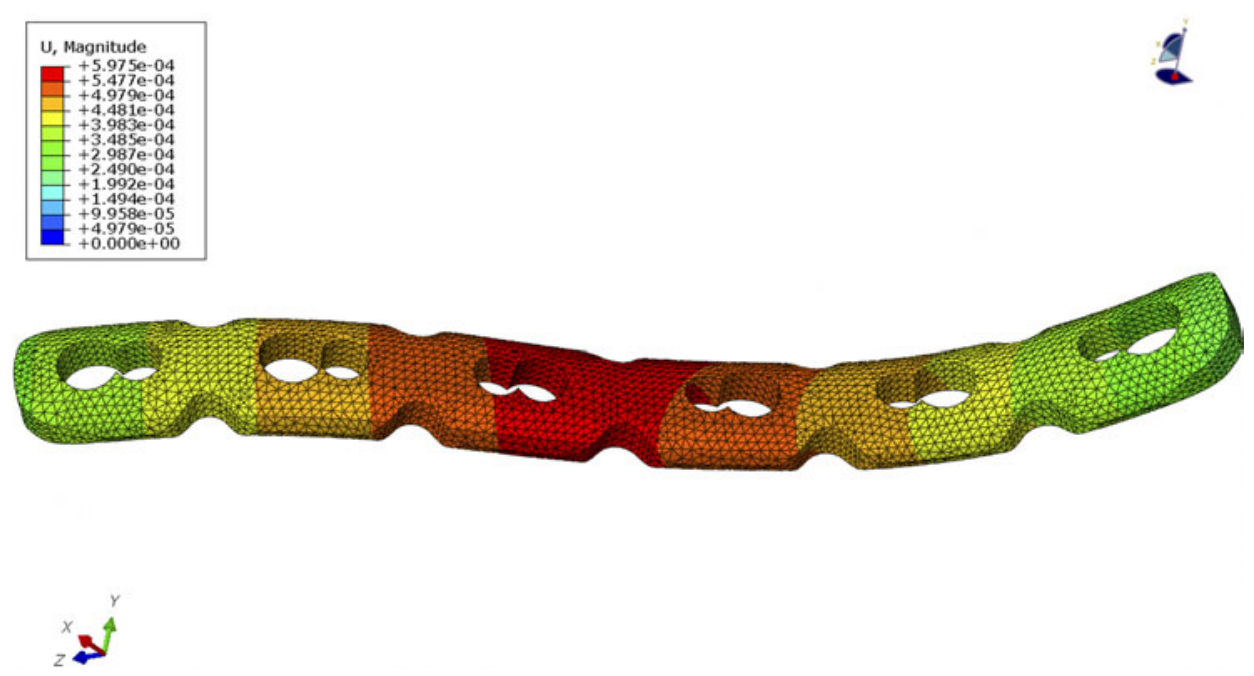

Figure 7. Displacements in the reconstruction plate. 


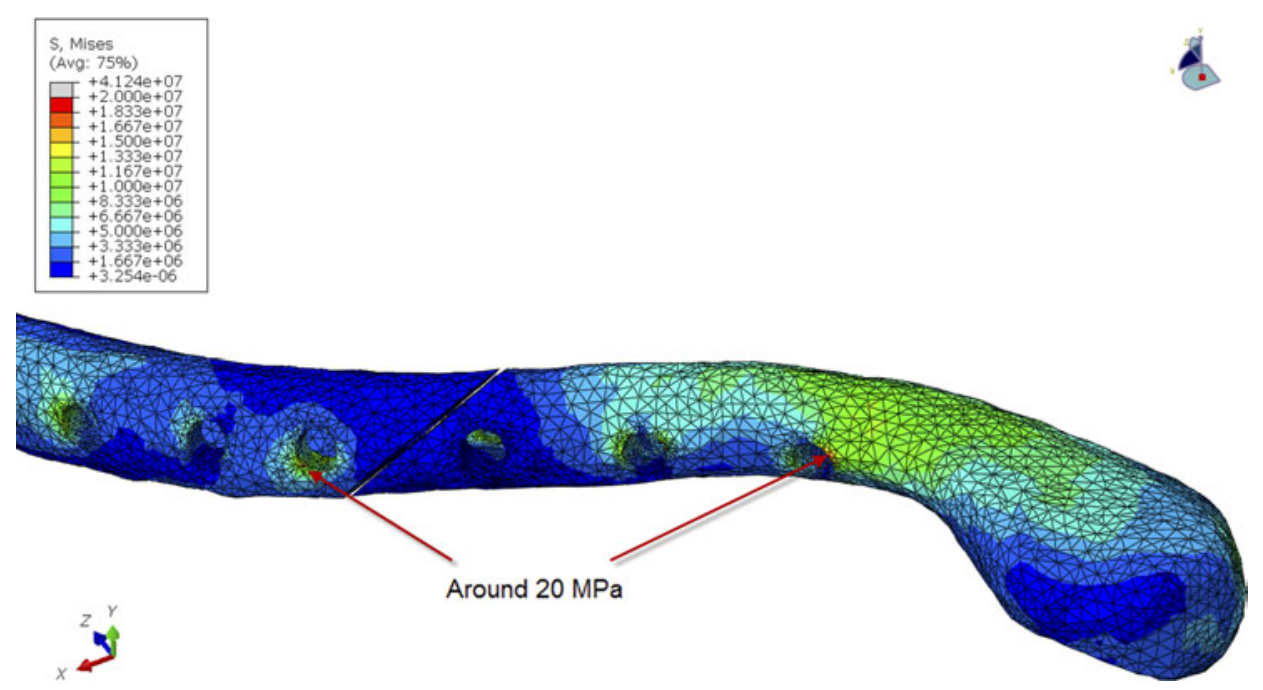

Figure 8. Stresses in bone with plate (the plate and screws are hidden in the picture).

Ataç et al. 2009; Lin et al. 2010), the femur (Peleg et al. 2006), the tibia (Raja Izaham et al. 2012) and for dental implants (Pérez et al. 2011).

The musculoskeletal model is based on rigid body dynamics meaning that it technically is constructed from a number of moving reference frames with mass and inertia properties and points and surfaces in these local reference frames forming joint centres to which muscles attach. The anatomical data, i.e. mass properties and local point coordinates, originate from published cadaver data. The bone geometries in the original musculoskeletal model only serve graphical purposes and have no influence on the computational model. These bone geometries originate from various sources, typically CT scans, and have in some cases been morphed to fit the point coordinates of the musculoskeletal model. These bone models are also relatively crude in the interest of computational performance.

Contrary to the musculoskeletal model, the FE model relies on the actual geometry of the clavicle bone and its accuracy is therefore more important in the FE model than in the multibody dynamics model. Consequently, the original clavicle bone from the musculoskeletal model was replaced by a new clavicle model of similar shape but with much higher resolution based on a CT-scan reconstruction. This new model was oriented to match the sternoclavicular and acromioclavicular joints. Small shape differences between the original and the new bone models mean that the muscle attachment points do not perfectly match the new bone. This has an unknown impact on the computational result, which can only be quantified by further analysis work. Alternatively, the muscle attachment

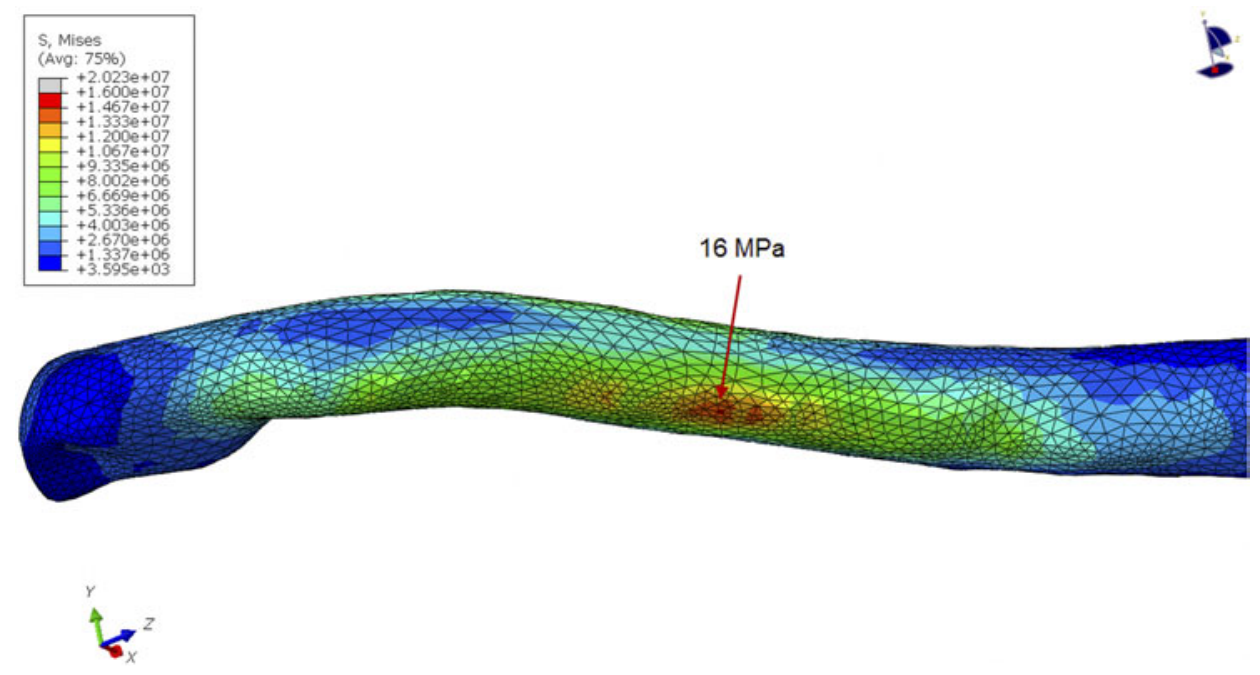

Figure 9. Stresses in bone without plate. 
points could be relocated to fit the new bone, but this strategy was discarded in the interest of preserving the original data-set, which is well-known and frequently used in the literature.

Earlier FE models in the literature, which contain a bone mesh generated from CT data, uses various methods to define the cortical section of the bone. Some are simplified with an even cortical thickness throughout the bone and some use the grey-scale values from the CT data as the basis for defining the cortical thickness. The best approximation of the bone materials properties is likely to be achieved by setting bone material properties which vary with the CT image density of the bone ( $\mathrm{Li}$ et al. 2012). At the present time, this was not compatible with importing the load case from the AnyBody software. In order to apply the load case to the STL mesh, the latter needs to be slightly rescaled and reoriented to match the biomechanical model. However, when applying the material properties based on the CT density, the STL mesh cannot be rescaled and must be in the origin position. Furthermore, since it turned out that the influence of the material definition in the inner part of the bone had small impact on the resulting stresses in the plate; it was assumed that detailed bone material definition is not the most crucial part in this type of study.

The stresses at the holes edges and the edge of the reconstruction segment were in the order of 400$460 \mathrm{MPa}$. The yield stress limit for the stainless steel (316 L) used in these plates differs between 190 and $690 \mathrm{MPa}$ depending on the model of plate used (Disegi 2009). These maximum stresses are local edge stresses which may seem high for lifting a load of $0.5 \mathrm{~kg}$; however, the forces on the clavicle from the muscle and ligaments in this position of the arm are in fact relatively high (Table 1) and hence will result in high stresses in a small reconstruction plate with tapering reconstruction segments. Furthermore, the fracture is in such a position that, together with a plate in current position, the present load case tends to open up the fracture and the fracture edges do not come into contact. The maximum stress in the bone without fracture, screw holes and plate was $16 \mathrm{MPa}$, and when the smallest cross-sectional area on the plate is subjected to the same load, it seems reasonable to assume that stresses in this magnitude will occur.

Based on the stress analysis of the plate, the areas exposed to the highest stresses are the edges of the holes and the reconstruction segments in the middle of the plate. According to these results, it is favourable to use a plate without reconstruction segments in the middle section. Furthermore, the plate could be designed with a reduced thickness at the outer ends, where the stresses are low, in order to make it smoother and less intrusive under the skin. As this is an initial model, which has not yet been validated, more work is needed before it can be used to draw applicable conclusions.
Integrating muscle forces in the FE analysis is a step towards more realistic simulations of clavicle plates. This combination of a multibody musculoskeletal analysis with an FE analysis of a bone with implant can be used to supplement physical and clinical testing for the comparison of various fixation methods for clavicle fractures. For example, it can be used initially when choosing what types of physical tests to perform. Other applications would be to simulate different types of fractures, various stages of the healing process, different loads and positions of the body, and combinations of the factors mentioned above. Hence, it can be used to study fixation methods, suitable body movements during rehabilitation, optimisation of new plates, verification of the strength of customised implants, etc.

As described in the methods chapter, the loading case $(\mathrm{xml})$ has to be combined with a mesh file (STL) to form the input file to the FE solver (at least while using a standard FE solver). A conclusion of the work is that this way of combining the bone mesh file (STL) with the loading case $(\mathrm{xml})$ and working with a so-called orphan mesh in the FE solver is time consuming and not suitable for quick analysis of many different fixation devises. After importing the mesh together with the muscle and ligament forces, the mesh should be replaced by a solid model of the bone, and then it is possible to create new screw holes in the solid model and hence quickly simulate different fixation devices and plate positioning. This will be a first part of the further work. Another important continuation of this work is to do more sensitivity analyses of the model and to compare stress distribution between superior and anterior plate position, plating with and without lag screw in the fracture gap and in plates with different shape. The screw-bone interaction is also interesting and could be modelled in better detail. It is especially interesting for the fixation of fractures in cancellous (osteoporotic) bone. That is usually not the case for midshaft clavicle fixation, but in other areas, primarily in proximity to joints, that is of high interest for the surgeons and will be dealt with in further similar studies.

\section{Acknowledgements}

The authors thank Joakim Asklund at Abaqus for the support with Abaqus CAE, the physicians Börje Samuelsson and Tryggve Ericsson at Östersund Hospital and the assistant tutors Lars-Erik Rännar and Mikael Bäckström, for their contribution to the study.

\section{Funding}

This work was funded by Swedish Agency for Economic and Regional Growth - Tillväxtverket, European Regional Development Fund.

\section{Notes}

1. Email: jr@m-tech.aau.dk

2. Email: mats.tinnsten@miun.se 


\section{References}

Alatamimi S, McKee M. 2008. Nonoperative treatment compared with plate fixation of displaced midshaft clavicular fractures. Surgical technique. J Bone Jt Surg (Am). 90 (2): $1-8$.

Arregui-Dalmases C, Pozo ED, Duprey S, Lopez-Valdes FJ, Lau A, Subit D, Kent R. 2010. A parametric study of hard tissue injury prediction using finite elements: consideration of geometric complexity, subfailure material properties, CTthresholding, and element characteristics. Traffic Inj Prev. 11(3):286-293.

Astier V, Thollon L, Arnoux PJ, Mouret F, Brunet C. 2008. Development of a finite element model of the shoulder: application during a side impact. Int J Crashworthiness. 13 (3):301-312.

Ataç MS, Erkmen E, Yücel E, Kurt A. 2008. Comparison of biomechanical behaviour of maxilla following Le Fort I osteotomy with 2- versus 4-plate fixation using 3D-FEA. Part 1: advancement surgery. Int $\mathbf{J}$ Oral Maxillofac Surg. 37 (12): $1117-1124$

Ataç MS, Erkmen E, Yücel E, Kurt A. 2009. Comparison of biomechanical behaviour of maxilla following Le Fort I osteotomy with 2- versus 4-plate fixation using 3D-FEA: Part 2: impaction surgery. Int $\mathrm{J}$ Oral Maxillofac Surg. 38 (1):58-63.

Bostman O, Manninen M, Pihljamaki H. 1997. Complications of plate fixation in fresh displaced midclavicular fractures. J Traum. 45(5):778-783.

Damsgaard M, Rasmussen J, Christensen ST, Surma E, de Zee M. 2006. Analysis of musculoskeletal systems in the AnyBody Modeling System. Simul Model Pract Theory. 14 (8):1100-1111.

Dines D, Lorich D, Helfet D. 2008. Solutions for complex upper extremity trauma. 1st ed. New York, NY: Thieme Medical Publishers, Inc.

Disegi J. 2009. Implant materials. Wrought $18 \%$ chromium-14\% nickel-2.5\% molubdenum stainless steel. 3rd ed. West Chester, NY: Synthes.

Golish SR, Oliviero J, Francke E, Miller M. 2008. A biomechanical study of plate versus intramedullary devices for midshaft clavicle fixation. J Orthop Surg Res. 3 (1):28-32.

Hill JM, McGuire MH, Crosby LA. 1997. Closed treatment of displaced middle-third fractures of the clavicle gives poor results. J Bone Jt Surg (Br). 79B(4):537-539.

Huang J, Toogood P, Wilber J, Cooperman D. 2007. Clavicular anatomy and the applicability of precontoured plates. J Bone Jt Surg (Am). 89(10):2260-2265.

Iannolo M, Werner FW, Sutton LG, Serell SM, VanValkenburg SM. 2010. Forces across the middle of the intact clavicle during shoulder motion. J Shoulder Elbow Surg. 19 (7):1013-1017.

Judd D, Pallis M, Smith E, Bottoni C. 2009. Acute operative stabilization versus nonoperative management of clavicle fractures. Am J Orthop. 38(7):341-345.
Kim S-H, Chang S-H, Son D-S. 2011. Finite element analysis of the effect of bending stiffness and contact condition of composite bone plates with simple rectangular cross-section on the bio-mechanical behaviour of fractured long bones. Compos B Eng. 42(6):1731-1738.

Kunze M, Schaller A, Steinke H, Scholz R, Voigt C. 2012. Combined multi-body and finite element investigation of the effect of the seat height on acetabular implant stability during the activity of getting up. Comput Methods Programs Biomed. 105(2):175-182.

Ledger M, Leeks N, Ackland T, Wang A. 2005. Short malunions of the clavicle: an anatomic and functional study. J Shoulder Elbow Surg. 14(4):349-354.

Li Z, Kindig MW, Kerrigan JR, Kent RW, Crandall JR. 2012. Development and validation of a subject-specific finite element model of a human clavicle. Comput Methods Biomech Biomed Eng. 16(8):1-11.

Lin C-L, Lin Y-H, Chang S-H. 2010. Multi-factorial analysis of variables influencing the bone loss of an implant placed in the maxilla: prediction using FEA and SED bone remodeling algorithm. J Biomech. 43(4):644-651.

McKee M, Wild L, Schemitsch E. 2003. Midshaft malunions of the clavicle. J Bone Jt Surg (Am). 85(5):790-797.

Nowak J. 2002. Clavicular fractures, epidemiology, union, malunion, nonunion. Uppsala: Acta Universitatis Upsaliensis.

Peleg E, Mosheiff R, Liebergall M, Mattan Y. 2006. A short plate compression screw with diagonal bolts - a biomechanical evaluation performed experimentally and by numerical computation. Clin Biomech. 21(9):963-968.

Pérez MA, Prados-Frutos JC, Bea JA, Doblaré M. 2011. Stress transfer properties of different commercial dental implants: a finite element study. Comput Methods Biomech Biomed Eng. 15(3):263-273.

Raja Izaham RMA, Abdul Kadir MR, Abdul Rashid AH, Hossain MG, Kamarul T. 2012. Finite element analysis of Puddu and Tomofix plate fixation for open wedge high tibial osteotomy. Injury. 43(6):898-902.

Renfree T, Conrad B, Wright T. 2010. Biomechanical comparison of contemporary clavicle fixation devices. J Hand Surg (Am). 35(4):639-644.

Taylor PR, Day RE, Nicholls RL, Rasmussen J, Yates PJ, Stoffel KK. 2011. The comminuted midshaft clavicle fracture: a biomechanical evaluation of plating methods. Clin Biomech. 26(5):491-496.

Wirtz DC, Schiffers N, Pandorf T, Radermacher K, Weichert D, Forst R. 2000. Critical evaluation of known bone material properties to realize anisotropic FE-simulation of the proximal femur. J Biomech. 33(10):1325-1330.

Wong C, Rasmussen J, Simonssen E, Hansen L, de Zee M, Dendorfer S. 2011. The influence of muscle forces on the stress didtribution in the lumbar spine. Open Spine J. 3:21-26.

Young P, Kennedy J, Cotton R. 2010. FEA of proximal humerus fracture with a fixation plate. Proceedings of the 2010 Simulia Customer Conference, 2010 May 25-27; RI, USA: Dassault Systemes. 